\title{
Manager Election of Power-Aware Wireless Sensor Networks Using Fuzzy Theory
}

\author{
Shu-Ching Wang, Shun-Sheng Wang*, \\ Chih-Ming Chang and Kuo-Qin Yan** \\ Department of Information Management, Chaoyang University of Technology, \\ 168, Jifong E. Rd., Wufong District, Taichung County 41349, Taiwan, ROC
}

(Received January 23, 2014; accepted March 13, 2014)

Key words: wireless sensor network, power-aware management, mobile agent

The power consumption rate and bandwidth of sensor nodes (SNs) in wireless sensor networks (WSNs) are important issues. For increasing the reliability of WSNs, in this paper, we propose a power-aware mechanism to select a stable cluster manager (CM) from SNs using fuzzy-based inference systems. Furthermore, our mechanism can trigger a mobile agent (MA) to distribute the managerial workload.

\section{Introduction}

Owing to the wide range of applications, the wireless sensor network (WSN) has rapidly become a popular mode.(1) However, the capability of a sensor node (SN) is limited by the computation, communication and power. Although relevant hardware technologies have been enhanced, the power constraints still present a challenge.(2) Thus, an efficient power-aware mechanism is necessary to avoid power exhaustion in WSNs.

However, efficient routing amongst a set of SNs is one of the most critical issues in WSNs. Therefore, the traditional routing protocols include a focus on the shortest path and cluster methods.(2) In the cluster method, the cluster manager (CM) can be elected to manage and forward messages for SNs within a specific range. ${ }^{(3)}$ Unfortunately, the topology of a WSN may be destroyed as the CM exhausts its limited power and bandwidth when overloaded with packets. To prolong the lifetime of a CM, a fuzzybased power-aware management system is proposed in this paper to assign the CM in a WSN. In addition, a multi-mobile agent (multi-MA) is used to assist the CM in managing the specific SNs when the workload is overloaded. However, our method is based on hierarchical cellular-based management (HCBM) proposed by Chang et al. ${ }^{(4)}$ and named power-aware HCBM.

*Corresponding author: e-mail: sswang@cyut.edu.tw

${ }^{* *}$ Corresponding author: e-mail: kqyan@cyut.edu.tw 


\section{Methods}

There are two phases in the power-aware HCBM: power-aware manager election (PME) phase and power-aware multi-MA assignation (PMAA) phase.

The main job of the PME phase is electing the appropriate CM for a WSN when a management overload exists. First, the information received by the GPS is used to select a CM for each cell. Subsequently, the distance, average roaming speed and power are considered. Finally, the power-aware assignation inference system (PAIS) is induced to select an appropriate CM.

The main task of the multi-MA is to assist in managing the SNs in intracells and divide into the management workload. The CM must elect the appropriate multi-MA by computing the capability of all SNs and assign the multi-MA via the PMAA phase where the congestion and misery index rises.

\section{Results}

\subsection{PME phase}

In the PME phase, there are two steps in electing the power-aware CM. (1) The cell is divided into several ranks and the $\mathrm{SN}$ is selected in the center of the cell according to the GPS information. (2) The average roaming speed and power of all SNs are computed to elect the appropriate $\mathrm{CM}$.

The location of each SN is compared to determine which SN can become the CM. However, to reduce the network load, the intracell is divided into three filter levels. The intracell is divided into six equal triangles based on the intracell center. The center in each triangle is connected to form a hexagon, which is $2 / 3$ multiples of the intracell of Level II. Level II is used to select the multi-MA. The cell closest to the center is Level I and the area is set up according to the density of the SN in the intracell. In general, the CM is closer to the center in the intracell than the Multi-MA; thus, Level I is smaller than Level II. We assume that $\mathrm{s}$ is a multiple of the maximum number of SNs. The area of Level I is $1 / s$ times that of Level II when the number of SNs is $s$ times to the preset threshold of SN. Namely, the area of Level I is $2 / 3 s$ times that of the intracell. However, the diagonal line of the hexagon formed by Level I cannot be less than $(r-0.86603) / 2$.

The divided filter is used to select the appropriate $\mathrm{SN}$ as the CM: thus, only the specific SNs need to participate in the PME phase. The CM is elected by $\min \left\{I_{\mathrm{u}}, 1 \leq u \leq\right.$ $n, u \in n, n$ is the amount of SNs $\}$. $I_{\mathrm{u}}$ represents the average roaming speed, power and distance from the center and shown in Eq. (1), where $M_{\mathrm{u}}$ is the average roaming speed, $T_{\mathrm{u}}$ is the power misery index, $D_{\mathrm{u}}=\left|d_{\mathrm{u}}-M\right|$ indicates the difference between $d_{\mathrm{u}}$ and $M, M$ is the threshold of connectivity, and $c_{1}, c_{2}$, and $c_{3}$ are the weights of each parameter.

$$
I_{\mathrm{u}}=c_{1} M_{\mathrm{u}}+c_{2} T_{\mathrm{u}}+c_{3} D_{\mathrm{u}}
$$

After the PME phase, a temporary power-aware CM in the intracell is elected. However, eq. (1) cannot fully represent the relationship among distance, roaming speed and power misery index. Therefore, the PAIS needs to be invoked into the PME phase to elect a more appropriate power-aware CM. The PAIS is divided into two steps: setting 
up the fuzzy members and fuzzy rule design.

Step 1: setting up fuzzy members. The fuzzy members include three parts: distance from center, roaming speed and power misery index. To reduce the complexity, the distance is divided into far and near. The roaming speed is divided into slow, medium and fast. The power misery index is divided into low, medium and high.

Step 2: fuzzy rules of CM. After Step 1, each SN transfers the information on the distance, roaming speed and power misery index to the temporary CM. Subsequently, the temporary $\mathrm{CM}$ selects an appropriate $\mathrm{CM}$. However, the weights of distance, roaming speed and power misery index are set to $0.7,0.2$, and 0.1 , respectively, as proposed by Huang et al.(5) Therefore, the weights of eq. (1) are adjusted to adapt to the WSN. The fuzzy rules contain 18 items and the lower sum has a higher probability of being the CM.

\subsection{PMAA phase}

There are two types of MA in the PMAA phase: the power-aware intra-MA (PIA-MA) and power-aware inter-MA (PIE-MA).

(1) PIA-MA: In general, the CM needs to detect where the bandwidth is overloaded and select the appropriate multi-MA during the PMAA phase. If the overload is coming from the SNs in the intracell, the CM will use the PAIS to elect the PIA-MA, which is located in Level II, to divide the management in the PMAA phase. The CM needs to manage the routing construction and packet relays between the intercells. The PIAMA is responsible for the other jobs such as identity management of SNs, routing construction and packet relay in the intracell.

(2) PIE-MA: The CM needs to elect a PIA-MA to divide jobs because of the overloading coming from SNs in the intracell. In contrast to the PIA-MA, the CM needs to elect a PIE-MA when the overload comes from the intercells. Therefore, the PIE-MA must be elected in Level I to determine the appropriate SNs. The PIE-MA is divided into two types of multi-MA according to their job characteristics. One is a transient PIE-MA, where the main job is providing the transmission service of when the CM is overloaded. The other is the permanent PIE-MA. The permanent PIE-MA needs to elect the new PIE-MA to replace it when the original permanent PIE-MA roams away from its intracell. In general, the CM can select numerous PIE-MAs to transfer the packets. However, the CM needs to handle the routing construction and packet relay in the intra/ intercell; hence, the power-aware multi-MA inference system (PMAIS) is provided.

To avoid the ping pong effect, ${ }^{(5)}$ the PMAIS uses a fuzzy theorem to determine whether the CM needs to elect a multi-MA or not. The PMAIS includes three steps.

Step 1: setting up the fuzzy member. Two factors are used to consider the status of the CM: throughput load and power misery index. To reduce the complexity, the throughput is divided into low $\left(\mathrm{L}_{\mathrm{t}}\right)$, medium $\left(\mathrm{M}_{\mathrm{t}}\right)$ and high $\left(\mathrm{H}_{\mathrm{t}}\right)$. However, the throughput load needs to be between $\left[0, t_{\max }\right]$. The power misery index is divided into low $\left(\mathrm{L}_{\mathrm{p}}\right)$, medium $\left(\mathrm{M}_{\mathrm{p}}\right)$, and high $\left(\mathrm{H}_{\mathrm{p}}\right)$, with the values between $\left[0, p_{\max }\right]$. The workload is divided into four states: low $\left(\mathrm{L}_{\mathrm{w}}\right)$, medium $\left(\mathrm{M}_{\mathrm{w}}\right)$, high $\left(\mathrm{H}_{\mathrm{w}}\right)$ and very high $\left(\mathrm{VH}_{\mathrm{w}}\right)$. Similarly, the values need to be between $\left[w_{\min }, w_{\max }\right]$. We set the membership function for the preset four states between $[0,1]$. 
Step 2: Constructing the fuzzy rule to trigger the multi-MA and fuzzy engine. The if-then fuzzy rules can be used to represent the workload state. Then, the management workload can be generalized.

Step 3: De-fuzzy. To obtain fast computation results, the CoA method ${ }^{(2)}$ is used to convert the fuzzy set representing the overall conclusion into a real number. On the basis of the conservative rule, the CM must trigger the multi-MA in the PMAA phase.

\section{Discussion}

Two assumptions are used in our experiments: (1) all SNs consume their power according to time and they do not charge their power even if they are in an idle state; and (2) there are seven cells in our simulated network topology and the elected CM is located in the center of the cells. On the basis of the assumptions, Network Simulation 2 (NS2) (6) is used to set a $100 \times 100 \mathrm{~m}^{2}$ topology and generate $20 \mathrm{SNs}$ randomly. Subsequently, JAVA is used to generate the PAIS and PMAIS. The packet sets are 512 bytes, using a constant bit rate (CBR) to transfer. The costs of transmission and receipt, which are computed using an energy consumption equation, NS2, are 0.6 , and $0.3 \mathrm{~A}$, respectively. In addition, to maintain objectivity, each experiment is simulated 500 times. There are four cases for our experiments.

Case 1: Node with a slow average roaming speed and sufficient power. The poweraware HCBM result is closer to the HCBM result before $15 \mathrm{~s}$. After $15 \mathrm{~s}$, the poweraware CM triggers the multi-MA to divide the load when it detects a VH load. However, this trigger action may increase the temporary CM load and stops after $20 \mathrm{~s}$. This brief workload increase will not have a serious effect on the CM lifetime; thus, the poweraware HCBM can still maintain the appropriate degree of power by which to decrease. The experiment result is shown in Fig. 1.

Case 2: Node with a fast average roaming speed and sufficient power. In Fig. 2, the power-aware HCBM gains showed a better result in lifetime than HCBM. It is because the nodes with the fast average roaming speed easily roam away from their original cell. Thus, packet loss and retransmission frequently occur. The CM easily exhausts its power and reduces the lifetime of the CM of the HCBM. However, the power-aware HCBM adjusts the weight to adapt to this network; thus, it can prolong the CMs' lifetime.

Case 3: Node with a slow average roaming speed and insufficient power. The CM easily exhausts its power in HCBM and power-aware HCBM. The power-aware HCBM has a longer lifetime than HCBM because the power-aware HCBM elects the appropriate $\mathrm{CM}$ and multi-MA to divide the workload. However, the results shown in Fig. 3 are worse than those in Cases 1 and 2. This is because the power is a critical issue in WSN for prolonging the CM lifetime. Obviously, the lifetime of WSN in the HCBM method almost stops at $20 \mathrm{~s}$.

Case 4: Node with a fast average roaming speed and insufficient power. Figure 4 shows the worst case. The $\mathrm{CM}$ easily roams out its section and exhausts its power. Therefore, the results of the HCBM and power-aware HCBM are the worst among all the types. However, the power-aware HCBM can elect the multi-MA to divide the workload; thus, it can obtain a better result than the HCBM. 


$$
\begin{aligned}
& I_{\mathrm{u} 1}=0.1 M_{\mathrm{u} 1}+0.1 T_{\mathrm{u} 1}+0.8 D_{\mathrm{u} 1} \\
& I_{\mathrm{u} 2}=0.2 M_{\mathrm{u} 2}+0.1 T_{\mathrm{u} 2}+0.7 D_{\mathrm{u} 2} \\
& I_{\mathrm{u} 3}=0.3 M_{\mathrm{u} 3}+0.1 T_{\mathrm{u} 3}+0.6 D_{\mathrm{u} 3} \\
& I_{\mathrm{u} 4}=0.4 M_{\mathrm{u} 4}+0.1 T_{\mathrm{u} 4}+0.5 D_{\mathrm{u} 4} \\
& I_{\mathrm{u} 5}=0.1 M_{\mathrm{u} 5}+0.2 T_{\mathrm{u} 5}+0.7 D_{\mathrm{u} 5} \\
& I_{\mathrm{u} 6}=0.1 M_{\mathrm{u} 6}+0.3 T_{\mathrm{u} 6}+0.6 D_{\mathrm{u} 6} \\
& I_{\mathrm{u} 7}=0.1 M_{\mathrm{u} 7}+0.4 T_{\mathrm{u} 7}+0.5 D_{\mathrm{u} 7}
\end{aligned}
$$

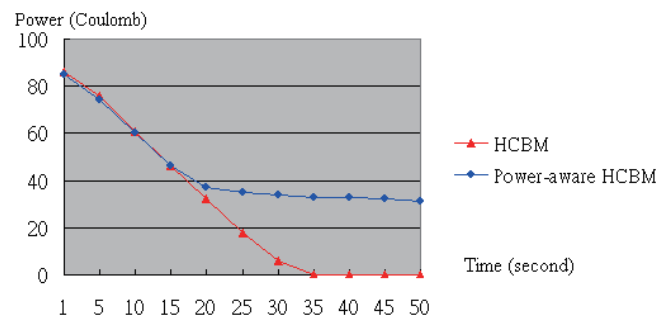

Fig. 1. (Color online) Result of experiment in case 1.

$$
\begin{aligned}
& I_{\mathrm{u} 1}=0.8 M_{\mathrm{u} 1}+0.1 T_{\mathrm{u} 1}+0.1 D_{\mathrm{u} 1} \\
& I_{\mathrm{u} 2}=0.7 M_{\mathrm{u} 2}+0.2 T_{\mathrm{u} 2}+0.1 D_{\mathrm{u} 2} \\
& I_{\mathrm{u} 3}=0.6 M_{\mathrm{u} 3}+0.3 T_{\mathrm{u} 3}+0.1 D_{\mathrm{u} 3} \\
& I_{\mathrm{u} 4}=0.5 M_{\mathrm{u} 4}+0.4 T_{\mathrm{u} 4}+0.1 D_{\mathrm{u} 4} \\
& I_{\mathrm{u} 5}=0.7 M_{\mathrm{u} 5}+0.1 T_{\mathrm{u} 5}+0.2 D_{\mathrm{u} 5} \\
& I_{\mathrm{u} 6}=0.6 M_{\mathrm{u} 6}+0.1 T_{\mathrm{u} 6}+0.3 D_{\mathrm{u} 6} \\
& I_{\mathrm{u} 7}=0.5 M_{\mathrm{u} 7}+0.1 T_{\mathrm{u} 7}+0.4 D_{\mathrm{u} 7}
\end{aligned}
$$

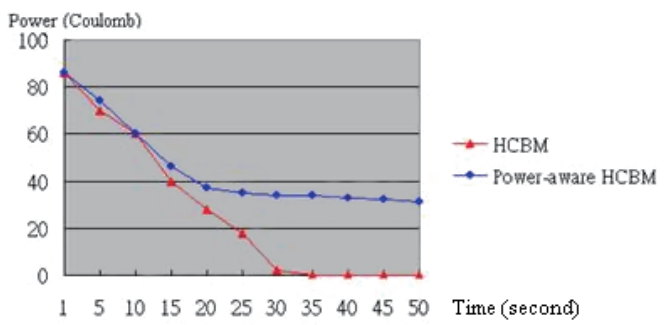

Fig. 2. (Color online) Result of experiment in case 2.

$$
\begin{aligned}
& I_{\mathrm{u} 1}=0.1 M_{\mathrm{u} 1}+0.8 T_{\mathrm{u} 1}+0.1 D_{\mathrm{u} 1} \\
& I_{\mathrm{u} 2}=0.2 M_{\mathrm{u} 2}+0.7 T_{\mathrm{u} 2}+0.1 D_{\mathrm{u} 2} \\
& I_{\mathrm{u} 3}=0.3 M_{\mathrm{u} 3}+0.6 T_{\mathrm{u} 3}+0.1 D_{\mathrm{u} 3} \\
& I_{\mathrm{u} 4}=0.4 M_{\mathrm{u} 4}+0.5 T_{\mathrm{u} 4}+0.1 D_{\mathrm{u} 4} \\
& I_{\mathrm{u} 5}=0.1 M_{\mathrm{u} 5}+0.7 T_{\mathrm{u} 5}+0.2 D_{\mathrm{u} 5} \\
& I_{\mathrm{u} 6}=0.1 M_{\mathrm{u} 6}+0.6 T_{\mathrm{u} 6}+0.3 D_{\mathrm{u} 6} \\
& I_{\mathrm{u} 7}=0.1 M_{\mathrm{u} 7}+0.5 T_{\mathrm{u} 7}+0.4 D_{\mathrm{u} 7}
\end{aligned}
$$

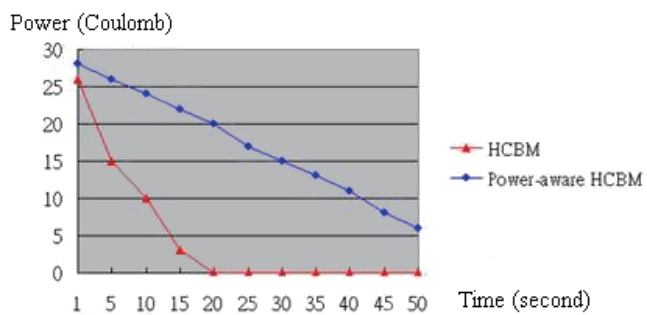

Fig. 3. (Color online) Result of experiment in case 3.

$$
\begin{aligned}
& I_{\mathrm{u} 1}=0.45 M_{\mathrm{u} 1}+0.45 T_{\mathrm{u} 1}+0.1 D_{\mathrm{u} 1} \\
& I_{\mathrm{u} 2}=0.40 M_{\mathrm{u} 2}+0.40 T_{\mathrm{u} 2}+0.2 D_{\mathrm{u} 2} \\
& I_{\mathrm{u} 3}=0.35 M_{\mathrm{u} 3}+0.35 T_{\mathrm{u} 3}+0.3 D_{\mathrm{u} 3}
\end{aligned}
$$

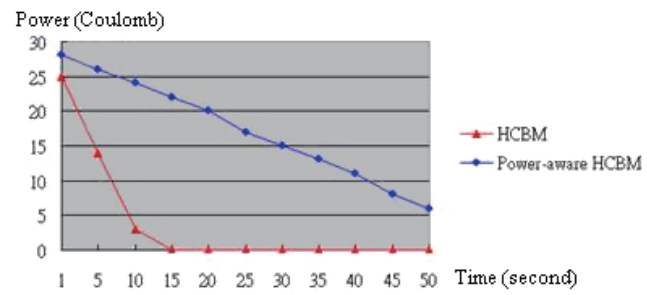

Fig. 4. (Color online) Result of experiment in case 4. 


\section{Conclusions}

In this study, inference engines of power-aware HCBM are used to elect a poweraware $\mathrm{CM}$ and multi-MA by considering the bandwidth and power misery index. In the PME phase, an appropriate power-aware CM is elected to take care of intracell and intercell tasks. In the PMAA phase, a multi-MA is used to divide the CM workload. Furthermore, there are two types of multi-MA, PIA-MA and PIE-MA. The PIA-MA is responsible for supporting the workload of the CM in intracell tasks and the PIE-MA is responsible for intercell tasks. Therefore, the CM lifetime can be prolonged in a WSN.

\section{References}

1 Y. C. Lee, H. Y. Lai and P. J. Lee: Int. J. Eng. Technol. Innovation 2 (2012) 283.

2 K. Q. Yan, S. C. Wang and Y. P. Tung: Commun. Int. Inf. Manage. Asso. J. 7 (2007) 17.

3 G. Indranil, D. Riordan and S. Srinivas: Proc. 3rd Annual Conf. on Commu. Netw. and Serv. Res. (IEEE, 2005) pp. 255-260.

4 C. Y. Chang and C. T. Chang: Comput. Commun. 24 (2001) 1554.

5 C. C. Huang, R. H. Chang and M. H. Guo: IEEE Wirel. Commun. Netw. 2 (2003) 1112.

6 The network simulator - NS-2, http://www.isi.edu/ nsnam/ns/ (accessed on Dec. 28, 2013). 